rev.relac.int.estrateg.segur.8(2):37-60,2013

\title{
EL MULTILATERALISMO AD HOC EN LAS RELACIONES CHINA-AMÉRICA LATINA Y EL CARIBE Y LAS COALICIONES SUR-SUR EN LAS NEGOCIACIONES DE LA OMC SOBRE EL COMERCIO AGRÍCOLA*
}

\author{
Natalia Andrea Lizama Poblete ${ }^{* *}$ \\ Yun-Tso Lee Cheng ${ }^{* * *}$
}

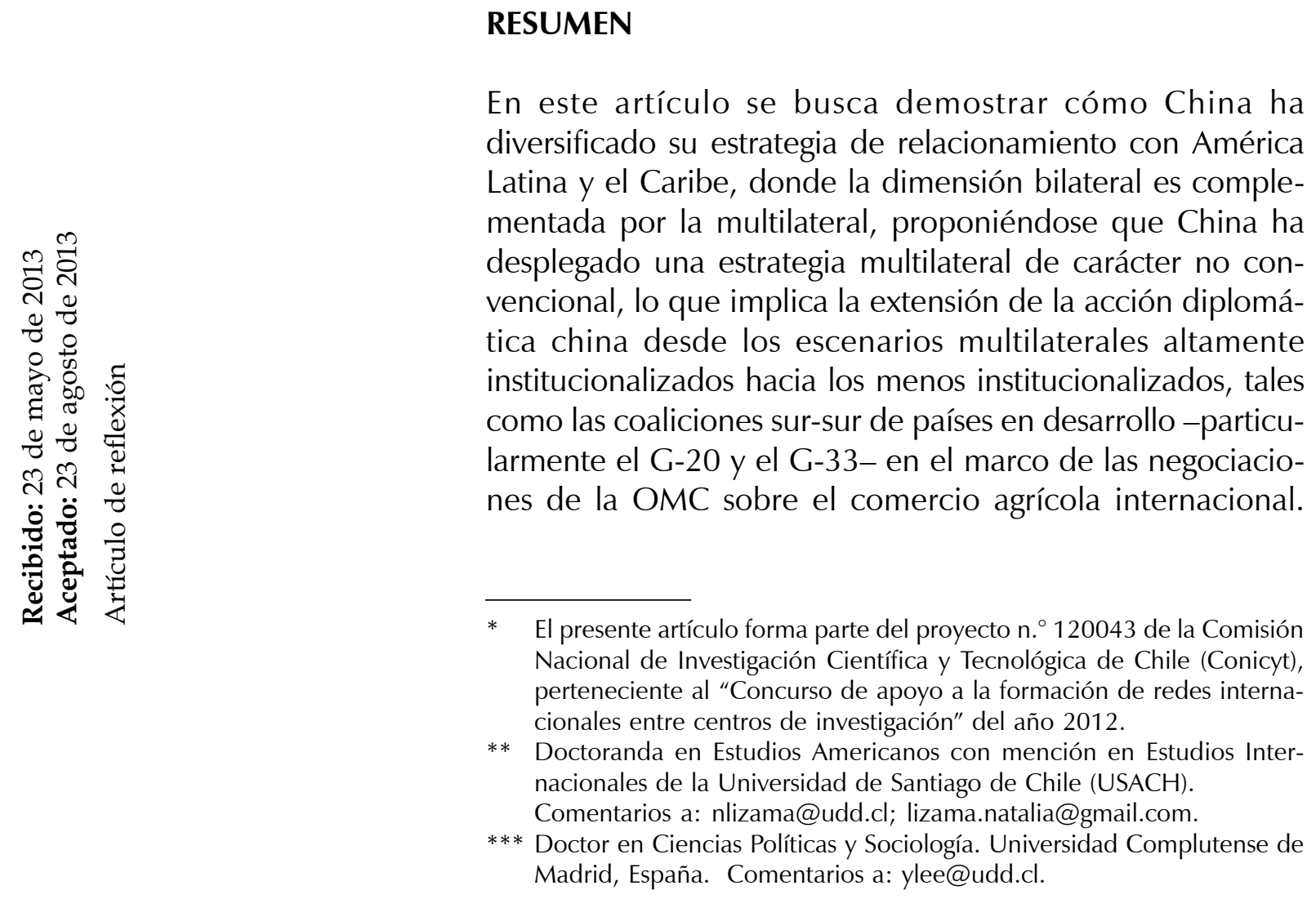


Ello responde a la composición de ambas coaliciones, sus intereses y objetivos, y su liderazgo colectivo gracias a la capacidad de consenso dentro de estas y junto a otras agrupaciones.

Palabras clave: China, América Latina y el Caribe, multilateralismo ad hoc, comercio agrícola, OMC.

\title{
MULTILATERALISM AD HOC IN THE RELATIONS CHINA-LATIN AMERICA AND THE CARIBBEAN AND SOUTH-SOUTH COALITIONS IN WTO'S NEGOTIATIONS REGARDING AGRICULTURAL TRADE
}

\begin{abstract}
This article aims to show how China has diversified its strategy of relations with Latin America and the Caribbean, where the bilateral dimension is complemented by a multilateral one, proposing that China has wielded a non-conventional strategy, which implies the growth of China's diplomatic action from highly institutionalized multilateral scenarios to other less institutionalized, such as the South-South coalitions of developing countries -specially the G-20 and the G-33- in the framework of WTO's international agricultural negotiations. This responds to the composition of both coalitions, their interests, objectives and their collective leadership thanks to the consensus-reaching capacity in these coalitions and along with others groups.
\end{abstract}

Keywords: China, Latin America and the Caribbean, multilateralism ad hoc, agricultural trade, WTO.

\section{O MULTILATERALISMO AD HOC NAS RELAÇÕES CHINA-AMÉRICA LATINA E O CARIBE E AS COLIGAÇÕES SUL-SUL NAS NEGOCIAÇÕES DA OMC SOBRE O COMÉRCIO AGRÍCOLA}

\section{RESUMO}

Neste artigo procura-se demonstrar como a China diversificou a sua estratégia de relações com a América Latina e o Caribe, na qual a dimensão bilateral é complementada pela multilateral e sugere que a China implantou uma estratégia multilateral de características não convencionais, que implica a extensão da ação diplomática chinesa a partir de cenários multilaterais altamente institucionalizados para os menos institucionalizados, tais como as coalizões Sul-Sul dos países em desenvolvimento, em particular no G-20 e G- 33, no âmbito das negociações da OMC sobre o comércio agrícola internacional. Isso responde a: a composição de ambas as coligações, seus interesses e objetivos e sua liderança coletiva graças à capacidade de consenso entre eles e com outros grupos.

Palavras-chave: China, América Latina e Caribe, multilateralismo ad hoc, comércio agrícola, OMC. 


\section{INTRODUCCIÓN}

En este artículo se pretende demostrar cómo China ha diversificado su estrategia de relacionamiento con América Latina y el Caribe, donde la dimensión bilateral es complementada por la multilateral, insertándose en coaliciones sur-sur, particularmente en aquellas que participan en las negociaciones internacionales sobre el comercio agrícola en el marco de las rondas de la Organización Mundial del Comercio (OMC), especialmente desde el ingreso de China a este organismo internacional en 2001.

Al respecto, se postula que, en su patrón de interacción con los países en desarrollo, particularmente con América Latina y el Caribe, China ha presentado un nuevo modelo de relacionamiento estratégico dentro del nivel multilateral, en consideración a la creciente participación de Beijing en organizaciones intergubernamentales de carácter ad hoc, informales y poco institucionalizadas, en particular aquellas conformadas por países en desarrollo, como las coaliciones sur-sur. Estos nuevos espacios multilaterales que van más allá de los escenarios tradicionales permiten canalizar intereses específicos -especialmente diseñados para Estados de menor poder- de un ámbito particular de la gobernanza global, que también es empleado por potencias emergentes asiáticas y latinoamericanas. En virtud de ello, se plantea que China ha desplegado una estrategia multilateral de carácter no convencional, denominada aquí "multilateralismo ad hoc", por su funcionalidad y su adaptabilidad a la diplomacia china, al facilitar el acercamiento y el diálogo cara a cara con sus aliados latinoamericanos en el marco de las negociaciones de la OMC sobre el comercio agrícola internacional, lo que permite diversificar su estrategia diplomática frente a los países latinoamericanos, extendiéndose desde lo bilateral hacia lo multilateral en instancias que le facilitan el ejercicio del poder blando chino.

Con este análisis se pretende abrir la discusión para indagar sobre la incursión de China en estos escenarios institucionales que, por su carácter ad-hoc y más bien informal, han generado menor atención en el estudio de las relaciones internacionales pero pueden, no obstante, dar luces acerca de la evolución de la diplomacia china durante los últimos años, desde su ingreso a la OMC en 2001, y abrir próximos planteamientos sobre la relevancia que estas instancias multilaterales de cooperación poseen para los países en desarrollo.

\section{MULTILATERALISMO DE CHINA Y AMÉRICA LATINA Y EL CARIBE EN COALICIONES SUR-SUR: EL G-20 Y EL G-33 EN LAS NEGOCIACIONES INTERNACIONALES DE LA OMC}

En consideración a los intentos de China por ingresar y posicionarse en espacios multilaterales de gobernanza global, gran parte de la literatura sobre el multilateralismo chino (Wu \& Lansdowe, 2008; Wang, 2000, y Zhu, 2010) aborda la política exterior china multilateral en organismos intergubernamentales formales y permanentes, descuidando las instancias internacionales más 
informales donde China también está reforzando su acción. Es posible plantear que el multilateralismo nominal (Wang, 2000) de la política exterior china no solo se debe concebir como el empleo de instituciones formales, como el Foro de Cooperación Económica Asia-Pacífico (APEC, por sus siglas en inglés) o la Asociación de Naciones del Sudeste Asiático (ASEAN, por sus siglas en inglés), entre otras, sino que, además, debe incorporarse a las organizaciones ad-hoc de carácter informal, como las coaliciones sur-sur, entre ellas las agrupaciones " $\mathrm{G}^{\text {"1 }}$ (o Ggroupings), particularmente en el contexto de las negociaciones agrícolas de la OMC.

Como se observa en el Cuadro 1, son variadas las coaliciones que participan activamente en las negociaciones comerciales de la OMC, tratando diversos asuntos, además de la agricultura. Entre ellas, se destaca no solo la participación de países latinoamericanos en diecinueve de las agrupaciones -con mayor o menor presencia, pues el número de países varía según el grupo-, sino que también se destaca la presencia de China en ocho de ellas, en las que comparte espacios con países latinoamericanos, como en las siguientes coaliciones: Miembros de reciente adhesión; G20 de países en desarrollo; G-33 de países en desarrollo; APEC; Países a los que se refiere el párrafo 6; Amigos de las Negociaciones Antidumping, y Patrocinadores del documento "W52".

Cuadro 1. Coaliciones que participan en las negociaciones comerciales de la OMC

\begin{tabular}{|l|c|c|c|c|}
\hline \multicolumn{1}{|c|}{ Coaliciones de la OMC } & $\begin{array}{c}\text { Temas } \\
\text { agrícolas }\end{array}$ & $\begin{array}{c}\text { Otros } \\
\text { temas }\end{array}$ & $\begin{array}{c}\text { Participa } \\
\text { China }\end{array}$ & $\begin{array}{c}\text { Participan } \\
\text { América } \\
\text { Latina y el } \\
\text { Caribe }\end{array}$ \\
\hline Grupo ACP & $X$ & & $X$ \\
Grupo Africano & $X$ & $X$ & $X$ & \\
Miembros en Desarrollo de Asia & $X$ & $X$ & $X$ \\
Unión Europea & $X$ & & $X$ \\
Mercosur & $X$ & & $X$ \\
G-90 & $X$ & & $X$ \\
Países Menos Adelantados (PMA) & $X$ & & $X$ \\
Economías pequeñas y vulnerables -agricultura & $X$ & $X$ & $X$ \\
Miembros de reciente adhesión & $X$ & & $X$ \\
\hline
\end{tabular}

1. Traducción propia de G-groupings.

2. Los otros temas corresponden a las siguientes cuestiones: generales, propiedad intelectual, normas para subvenciones a la pesca, normas antidumping y registro de indicaciones geográficas ADPIC. Véase Organización Mundial del Comercio (OMC). (n. d.). "Grupos en el marco de las negociaciones". Extraído abril 30, 2013, desde http:// www.wto.org/spanish/tratop_s/dda_s/negotiating_groups_s.htm. 


\begin{tabular}{|c|c|c|c|c|}
\hline Economías en transición de ingreso bajo & $X$ & & & \\
\hline Grupo de Cairns & $X$ & & & $X$ \\
\hline Grupo de Productos Tropicales & $X$ & & & $X$ \\
\hline G-10 & $X$ & & & \\
\hline G-20 & $X$ & & $X$ & $X$ \\
\hline G-33 & $X$ & & $X$ & $X$ \\
\hline Los "Cuatro del Algodón" & $X$ & $X$ & & \\
\hline APEC & & $X$ & $X$ & $X$ \\
\hline ASEAN & & $X$ & & \\
\hline $\begin{array}{l}\text { Economías pequeñas y vulnerables - acceso a los } \\
\text { mercados para los productos no agrícolas (AMNA) }\end{array}$ & & $X$ & & $X$ \\
\hline Economías pequeñas y vulnerables - normas & & $X$ & & $X$ \\
\hline Grupo AMNA 11 & & $X$ & & $x$ \\
\hline Países a los que se Refiere el Párrafo 6 & & $X$ & $X$ & $X$ \\
\hline Amigos de la Ambición (AMNA) & & $X$ & & \\
\hline Amigos de las Negociaciones Antidumping & & $X$ & $X$ & $x$ \\
\hline Amigos de los Peces & & $X$ & & $x$ \\
\hline Patrocinadores del documento "W52" & & $X$ & $X$ & $x$ \\
\hline Propuesta conjunta (Propiedad intelectual) & & $X$ & & $X$ \\
\hline
\end{tabular}

Fuente: elaboración propia a partir de Organización Mundial del Comercio (OMC). (n. d.). "Grupos en el marco de las negociaciones". Extraído abril 30, 2013, desde http://www.wto.org/spanish/tratop_s/dda_s/negotiating groups_s.htm; y Organización Mundial del Comercio (OMC). (n. d.). "Grupos en las negociaciones sobre la agricultura". Extraído abril 30, 2013, desde http://www.wto.org/spanish/tratop_s/agric_s/negoti_groups_s.htm.

En materia agrícola, en general, los intereses de China en el marco de la OMC se centran en lograr una "robusta liberalización de los tres 'pilares' de las negociaciones agrícolas de la OMC: acceso a los mercados, reducción de los subsidios a las exportaciones y menor ayuda interna" (Rosen, Rozelle \& Huang, 2004: 2). Recordemos que China ha experimentado casi cuatro décadas de reforma de su economía, desde una dominada por el Estado a una regida por el mercado; en este contexto, el ingreso de China a la OMC en 2001 obedeció a la necesidad de estabilizar el acceso al mercado, asegurando un mercado externo abierto a las exportaciones chinas (Scott \& Wilkinson, 2011).

En atención a los intereses de China en el ámbito del comercio agrícola internacional, y al marco general de inserción de China en las coaliciones de la $\mathrm{OMC}$, se sostiene que en estos nuevos 
espacios diplomáticos de organizaciones intergubernamentales no convencionales, particularmente las coaliciones G-20 y G-33, la participación de China se explica por tres condicionantes: la composición de ambas coaliciones, los intereses y objetivos que estas persiguen, y el liderazgo colectivo que han desarrollado dentro de los procesos de negociación institucional y que les ha permitido presentar propuestas sobre las reformas a las modalidades del comercio agrícola internacional en sus tres pilares: acceso al mercado, ayuda interna y subvenciones a la exportación, en virtud de la capacidad de consenso tanto dentro de otras agrupaciones como junto a ellas.

\subsection{COMPOSICIÓN DEL G-20 Y EL G-33}

El G-33 de países en desarrollo, que también recibe el nombre de Amigos de los Productos Especiales, se formó en la Quinta Conferencia Ministerial de la OMC, celebrada en septiembre de 2003 en Cancún, México, como una alianza por los productos especiales (PE) y un mecanismo de salvaguardia especial (MSE). Su principal objetivo consiste en lograr que el sistema de la OMC entregue mayor flexibilidad a los países en desarrollo para que puedan efectuar una apertura limitada de sus mercados agropecuarios. ${ }^{3}$ Está formado por Antigua y Barbuda; Barbados; Belice; Benín; Bolivia; Botsuana; Congo; Cuba; Costa de Marfil; Dominica; El Salvador; Filipinas; Granada; Guatemala; Guyana; Haití; Honduras; India; Indonesia; Jamaica; Kenia; Madagascar; Mauricio; Mongolia; Mozambique; Nicaragua; Nigeria; Pakistán; Panamá; Perú; República Dominicana; República de Corea; San Cristóbal y Nieves; San Vicente y las Granadinas; Santa Lucía; Senegal; Sri Lanka; Surinam; Tanzania; Trinidad y Tobago; Turquía; Uganda; Venezuela; Zambia, Zimbabue y China.

Por su parte, el G-20 de países en desarrollo se estableció también en el marco de la Quinta Conferencia Ministerial de la OMC realizada en Cancún y está conformado por veintitrés países en desarrollo: China; India; Indonesia; Pakistán; Tailandia; Filipinas; Argentina; Bolivia; Brasil; Chile; Cuba; Ecuador; Guatemala; México; Paraguay; Perú; Uruguay; Venezuela; Egipto; Nigeria; Sudáfrica, Tanzania y Zimbabue.

Si se analiza la membrecía de ambas coaliciones y se consideran solo los países latinoamericanos que las conforman, como se expone en el Cuadro 2, se puede notar que las dos reúnen a casi toda América Latina y el Caribe, incluido México, con la excepción de Costa Rica y algunos Estados caribeños. En efecto, el G-20 incluye a los países de Sudamérica que no hacen parte del G-33, mientras que el G-33 incorpora a países de Centroamérica y el Caribe que el G-20 no abarca. Ahora bien, según la adhesión institucional de los países latinoamericanos a organismos

3. Véase Organización Mundial del Comercio (OMC). (n. d.). "Grupos en las negociaciones sobre la agricultura". Extraído abril 30, 2013, desde http://www.wto.org/spanish/tratop_s/agric_s/negoti_groups_s.htm. 
de integración regionales, podemos notar que unidas, y con excepción de Costa Rica, ambas coaliciones reúnen a casi todo el Sistema de la Integración Centroamericana (SICA), a gran parte de la Comunidad del Caribe (Caricom), al Mercado Común del Sur (Mercosur), a la Unión de Naciones Suramericanas (Unasur) y a la Comunidad de Estados Latinoamericanos y Caribeños (Celac). De esta manera, se entiende la participación de China en ambas, en lo que concierne a su estrategia de asociación estratégica con América Latina y el Caribe; ambas coaliciones son escenarios clave para la diplomacia multilateral ad hoc de China con la región.

Cuadro 2. Membrecía latinoamericana del G-20 y el G-33

\begin{tabular}{|c|c|c|c|}
\hline Coalición & Sudamérica & Centroamérica & Caribe \\
\hline G-20 & $\begin{array}{l}\text { Argentina } \\
\text { Bolivia } \\
\text { Brasil } \\
\text { Chile } \\
\text { Ecuador } \\
\text { Paraguay } \\
\text { Perú } \\
\text { Uruguay } \\
\text { Venezuela }\end{array}$ & $\begin{array}{l}\text { Guatemala } \\
\text { México }^{4}\end{array}$ & Cuba \\
\hline G-33 & $\begin{array}{l}\text { Bolivia } \\
\text { Guyana } \\
\text { Surinam } \\
\text { Perú } \\
\text { Venezuela }\end{array}$ & $\begin{array}{l}\text { Belice } \\
\text { El Salvador } \\
\text { Nicaragua } \\
\text { Panamá } \\
\text { Honduras } \\
\text { Guatemala }\end{array}$ & $\begin{array}{l}\text { Antigua y Barbuda } \\
\text { Barbados } \\
\text { Cuba } \\
\text { Dominica } \\
\text { Granada } \\
\text { Haití } \\
\text { Jamaica } \\
\text { República Dominicana } \\
\text { San Cristóbal y Nieves } \\
\text { San Vicente y las Granadinas } \\
\text { Santa Lucía } \\
\text { Surinam } \\
\text { Trinidad y Tobago } \\
\text { Venezuela }\end{array}$ \\
\hline
\end{tabular}

4. Solo para efectos de orden del Cuadro, se incluye a México como parte de Centroamérica. 
En el caso particular del G-20, y en lo relativo a su composición, cabe subrayar ciertos aspectos llamativos que ayudan a comprender la presencia de China en esta coalición. En este grupo participan, junto con China, potencias medianas emergentes clave, como India y Brasil, que han desplegado una influyente diplomacia de mediana potencia en otros ámbitos de la política internacional. El resto de la coalición está integrado por países considerados también como potencias medianas, tales como Egipto, Nigeria, Sudáfrica, Indonesia, Pakistán, México, Argentina, Chile y Venezuela. Los otros miembros pueden considerase como potencias pequeñas.

De acuerdo al grado de desarrollo de sus mercados, cabe destacar de los miembros del G-20 que un importante número de ellos se puede catalogar incluso como "mercados emergentes": Egipto, Sudáfrica, Indonesia, Pakistán, Filipinas, Tailandia, Argentina, Brasil, Chile, México, China, India y Perú, algunos de los cuales -como Brasil, China, India, Egipto, Indonesia, México, Sudáfrica y Filipinas- son denominados "grandes mercados emergentes". Actualmente, "las economías emergentes [...] representan el grueso del crecimiento del producto interno bruto (PIB) mundial y una porción significativa del comercio internacional" (Rosales, 2009: 78).

Adicionalmente, el G-20 comprende una considerable parte de la población y de la producción y el comercio agrícola mundiales. Según el informe de los dos primeros años de actividades del G-20 publicado en 2005, los miembros de esta coalición abarcan el $63 \%$ de todos los agricultores, el $51 \%$ de la población mundial, el $20 \%$ de la producción agrícola, el $26 \%$ del total de las exportaciones agrícolas y el $17 \%$ de las importaciones de productos agrícolas mundiales (G-20 de países en desarrollo, 2005). ${ }^{5}$

En el caso del G-33, se resalta el hecho de que, a pesar de que todos los Estados que componen esta coalición son países en desarrollo que poseen, en general, un ingreso medio, el grupo es bastante diverso debido a que incluye a una potencia emergente que es a la vez el productor agrícola más grande (China), y al productor agrícola más pequeño (Granada), así como también a uno de los países en desarrollo más avanzados (Corea del Sur) y a uno de los países más pobres del mundo, Haití (Jales, 2005). Por lo demás, sus países miembros poseen vulnerabilidades específicas, a partir de lo cual el grupo defiende fuertemente los productos especiales y el mecanismo de salvaguardia especial.

Esta somera descripción geopolítica de la composición de las coaliciones nos permite notar el grado de diversidad presente en ambas agrupaciones, respecto del poder relativo que poseen sus miembros, puesto que países muy pequeños, como los del Caribe, y otros que son catalogados como potencias medianas emergentes comparten la misma estructura organizacional. No obstante, un análisis de cluster efectuado en Constantini et al. (2007) entrega luces más bien

5. G-20 de países en desarrollo (2005). Two Years of Activities of the G-20: Moving Forward the Doha Round. Extraído diciembre 5, 2009, desde http://www.g-20.mre.gov.br.

EL MULTILATERALISMO AD HOC EN LAS RELACIONES CHINA-AMÉRICA LATINA Y EL CARIBE Y LAS COALICIONES SUR-SUR EN LAS NEGOCIACIONES DE LA OMC SOBRE EL COMERCIO AGRÍCOLA 
respecto de los efectos de una cierta similitud que existe entre el G-20 y el G-90, en atención a que dentro del G-90 figura gran parte de los miembros del G-33, a la vez que complementa el anterior análisis geopolítico al ayudar a explicar la ubicación de China en ambas coaliciones.

Este análisis de cluster es aplicado a algunas de las coaliciones de negociación que participan en las negociaciones de la OMC (G-20, G-90, G-10, Cairns, UE-25 y Estados Unidos) sobre la base de las siguientes variables relativas al nivel de desarrollo económico, social e institucional; la apertura al comercio; la estructura agrícola y las políticas de acceso a los mercados, retratando así las características estructurales de una coalición. Este análisis permite una mejor comprensión de la posición relativa de los diferentes grupos y, en ese sentido, parte de sus conclusiones señala que el G-20 y el G-90 están "globalmente 'cercanos' uno del otro" y distantes de otros grupos (Constantini et al., 2007: 877). Según el estudio, el G-90 está formado por dos clusters, que aparecen muy cercanos entre sí, mientras que el G-20 está compuesto por un tercer cluster que es el cluster más próximo a los dos del G-90. Tal como afirma el estudio, "esto sugiere la posibilidad de definir algunas posiciones comunes en las negociaciones, creando un frente amplio de países en desarrollo que equilibran el poder político y económico de los países desarrollados" (Constantini et al., 2007: 877). Ello permite comprender por qué China escogió participar en el G-33 y el G-20 sobre la base del grado 'similitud' respecto de un conjunto de variables relacionadas con la estructura socioeconómica de los miembros de ambos grupos.

\subsection{INTERESES Y OBJETIVOS DEL G-20 Y EL G-33}

En general, ambas agrupaciones de países en desarrollo buscan lograr mejores condiciones para el comercio de productos agrícolas para los tres pilares en los que se concentran las negociaciones: acceso a los mercados, ayuda interna y subvención a las exportaciones. Siguiendo las categorías de las diferentes formas de asociación informal entre Estados, ambas coaliciones coinciden con las llamadas single-based coalitions o, también, issue-based coalitions (o "coaliciones basadas en un tema"), lo que significa que se abocan a defender intereses pertenecientes a un sector del comercio internacional, que en este caso es la agricultura.

Específicamente, las preocupaciones del G-33 se focalizan en que la subsistencia de la población dedicada a la agricultura podría estar amenazada por una mayor liberalización, especialmente si los subsidios se mantuvieran y los agricultores tuvieran que competir con importaciones artificialmente abaratadas. En concordancia con ello, el objetivo del G-33 es garantizar la seguridad alimentaria, los medios de subsistencia y el desarrollo rural de los agricultores pequeños, vulnerables y pobres, lo que constituye los cimientos centrales de la agenda de desarrollo de los países del G-33 (G-33 de países en desarrollo, 12 de junio de 2005), ${ }^{6}$ debido a que, según han

6. G-33 de países en desarrollo. (2005). G-33 Ministerial Communiqué, Jakarta, 12 June 2005. Extraído marzo 20, 2013, desde http://www.iatp.org/files/451_2_73101.pdf. 
manifestado sus miembros, "el problema de la seguridad alimentaria y la subsistencia, así como el desarrollo rural, constituyen una expresión concreta del derecho de los países en desarrollo al desarrollo y, por lo tanto, ello exige una solución comprehensiva en los tres pilares de las negociaciones agrícolas" (G-33 de países en desarrollo, 12 de junio de 2005: 1).7

Por eso, un objetivo es extender las flexibilidades a los países en desarrollo (G-33 de países en desarrollo, 12 de junio de 2005) ${ }^{8}$ a fin de proteger a sus agricultores por intermedio de aranceles más altos para productos especiales y un nuevo mecanismo de salvaguardia especial para los países en desarrollo. Asimismo, consideran que "se debería incluir en todos los elementos de las negociaciones un trato especial y diferenciado más significativo y operacional para los países en desarrollo" (G-33 de países en desarrollo, 12 de junio de 2005: 1). ${ }^{9}$

Por otra parte, el principal elemento de la agenda del G-20 es presionar para eliminar los subsidios a las exportaciones agrícolas y lograr reducciones sustanciales en la ayuda interna que distorsiona el comercio. Desde su formación en 2003, el G-20 acogió los intereses del mundo en desarrollo cuando criticó el documento común de la Unión Europea y Estados Unidos sobre agricultura presentado en agosto de 2003, que "buscaba limitar las discusiones a fin de establecer un mero 'marco' para las negociaciones agrícolas a solo unas cuantas semanas de la Reunión Ministerial de Cancún" (Ismail, 2007). En opinión de los promotores del G-20, el texto implicaba marginar los intereses de los países en desarrollo y podría generar una polarización en el área agrícola, afectando las metas de la Agenda de Desarrollo de Doha, lo que finalmente impulsó a los países en desarrollo a unir fuerzas para oponerse a un texto altamente insatisfactorio (Da Motta Veiga, 2005). Debido al innegable vínculo entre la agricultura y el desarrollo, el G-20 estimó -en el comunicado ministerial publicado el 9 de septiembre de 2003 en Cancún- que "la agricultura debería incorporarse en su totalidad en las reglas del sistema comercial multilateral, con vistas a eliminar las distorsiones que prevalecen en el comercio y la producción agrícolas" (G-20 de países en desarrollo, 2005: 3). ${ }^{10}$ Brasil e India elaboraron una propuesta que rápidamente ganó el respaldo de otros países en desarroIlo, como China, Sudáfrica, Indonesia, Filipinas y Argentina, parte de la coalición que se denominaría G-20 y se constituiría con la finalidad de avanzar en la liberalización y reforma de la agricultura de los países desarrollados, sobre la base de la justicia social y el desarrollo de los países en desarrollo (Ismail, 2007).

7. G-33 de países en desarrollo. (2005). G-33 Ministerial Communiqué, Jakarta, 12 June 2005. Extraído marzo 20, 2013, desde http://www.iatp.org/files/451_2_73101.pdf.

8. G-33 de países en desarrollo. (2005). G-33 Ministerial Communiqué, Jakarta, 12 June 2005. Extraído marzo 20, 2013, desde http://www.iatp.org/files/451_2_73101.pdf.

9. G-33 de países en desarrollo. (2005). G-33 Ministerial Communiqué, Jakarta, 12 June 2005. Extraído marzo 20, 2013, desde http://www.iatp.org/files/451 2 73101.pdf.

10. G-20 de países en desarrollo (2005). Two Years of Activities of the G-20: Moving Forward the Doha Round. Extraído diciembre 5, 2009, desde http://www.g-20.mre.gov.br. 
Como se ha señalado, las coaliciones analizadas han presentado propuestas tendientes a reformar las modalidades del comercio agrícola internacional en sus tres pilares: acceso a los mercados, ayuda interna y subsidios a las exportaciones, lo cual pasa a detallarse a continuación.

\section{Primer pilar: acceso a los mercados ${ }^{11}$}

En relación con este pilar, el G-33 ha manifestado sostenidamente su posición respecto de las modalidades de acceso a los mercados, estableciendo como grupo central a los pequeños agricultores y las poblaciones rurales desfavorecidas, y planteando propuestas relativas a los instrumentos de trato especial y diferenciado, tales como el mecanismo de salvaguardia especial y los productos especiales.

En sus declaraciones oficiales, el G-33 ha manifestado que el mecanismo de salvaguardia especial debe, a su juicio, proporcionar "un remedio efectivo para los países en desarrollo frente al aumento de las importaciones y el descenso de los precios" (G-33 de Países en Desarrollo, 12 de junio de 2005: 3). ${ }^{12} \mathrm{Al}$ tratarse de un mecanismo de salvaguardia para los países en desarroIlo, "el mecanismo de salvaguardia especial debe ser capaz de responder a las necesidades y circunstancias particulares de los distintos países en desarrollo y países menos desarrollados". (G-33 de países en desarrollo, 12 de junio de 2005: 3). ${ }^{13}$

Sobre los productos especiales, los miembros del G-33 sostienen que deben tener garantizado el acceso al MSE y ser eximidos de las reducciones arancelarias y de cualquier compromiso relativo a los contingentes arancelarios (G-33 de Países en Desarrollo, 12 de junio de 2005). ${ }^{14}$ Los productos que se deben designar como productos especiales deben ser aquellos que cumplan el criterio de seguridad alimentaria, seguridad de subsistencia y desarrollo rural. En consecuencia, los miembros del G-33 "acentúan el hecho de que la designación del producto especial debería reflejar la evaluación individual de los países respecto de la importancia de productos especiales para la seguridad alimentaria y de subsistencia, y el desarrollo rural" (G-33 de Países en Desarrollo, 12 de junio de 2005: 2). ${ }^{15}$ Junto con el MSE, los productos especiales "son fundamentales para cualquier operacionalización

11. Para obtener mayores detalles sobre los aspectos que cubre el pilar dedicado al acceso a mercados, véase Organización Mundial del Comercio (OMC). (n. d.). "Agricultura: mercados más equitativos para los agricultores". Extraído marzo 25, 2013, desde http://www.wto.org/spanish/thewto_s/whatis_s/tif_s/agrm3_s.htm.

12. G-33 de Países en Desarrollo. (2005). G-33 Ministerial Communiqué, Jakarta, 12 June 2005. Extraído marzo 20, 2013, desde http://www.iatp.org/files/451_2_73101.pdf.

13. G-33 de Países en Desarrollo. (2005). G-33 Ministerial Communiqué, Jakarta, 12 June 2005. Extraído marzo 20, 2013, desde http://www.iatp.org/files/451_2_73101.pdf.

14. G-33 de Países en Desarrollo. (2005). G-33 Ministerial Communiqué, Jakarta, 12 June 2005. Extraído marzo 20, 2013, desde http://www.iatp.org/files/451_2 73101.pdf.

15. G-33 de Países en Desarrollo. (2005). G-33 Ministerial Communiqué, Jakarta, 12 June 2005. Extraído marzo 20, 2013, desde http://www.iatp.org/files/451_2_73101.pdf. 
significativa del trato especial y diferenciado, y cruciales para abordar la seguridad alimentaria y de subsistencia, así como las necesidades de desarrollo rural de los países en desarrollo" (G-33 de Países en Desarrollo, 12 de junio de 2005: 2). ${ }^{16}$ En este sentido, para el G-33 lo central del pilar de acceso a los mercados es la fórmula de la reducción arancelaria, que "puede ayudar en gran medida al esfuerzo de los países en desarrollo para salvaguardar la seguridad alimentaria y de subsistencia y el desarrollo rural" (G-33 de Países en Desarrollo, 12 de junio de 2005: 3). ${ }^{17}$

En 2008, el G-33 expuso su preocupación y su desacuerdo respecto del texto de modalidades de diciembre de ese año, mediante una propuesta presentada el 27 de enero de 2010 (Organización Mundial del Comercio, 28 de enero de 2010),$^{18}$ donde "cuestiona los criterios del MSE y afirma que lo que permanece en la mesa de negociación no tiene mayor incidencia en la eliminación de las distorsiones en el mercado agrícola" (International Centre for Trade and Sustainable Development, febrero de 2010: 1). ${ }^{19}$ La propuesta del G-33 parte de la base de que dicho mecanismo no sea visto a través del prisma del comercio, en atención a "la realidad objetiva de la agricultura en la mayor parte del mundo en desarrollo, donde la preocupación central de cientos de millones de personas dedicadas a la agricultura es la supervivencia, no el comercio" (Organización Mundial del Comercio, 28 de enero de 2010: 2). ${ }^{20}$ Por lo tanto, a juicio del Grupo, la perspectiva correcta es ver el MSE como "un instrumento que permita a los países en desarrollo abordar sus preocupaciones centrales de seguridad alimentaria y de subsistencia y desarrollo rural, a la vez que asumir los compromisos tendientes a la liberalización" (Organización Mundial del Comercio, 28 de enero de 2010: 2). ${ }^{21}$

En opinión del G-33, la nueva conceptualización del MSE debe "permitir que los países en desarrollo impongan medidas de salvaguardia a las importaciones cuando se presente un aumento en las importaciones o un brusco descenso en los precios" (International Centre for Trade

16. G-33 de Países en Desarrollo. (2005). G-33 Ministerial Communiqué, Jakarta, 12 June 2005. Extraído marzo 20, 2013, desde http://www.iatp.org/files/451_2_73101.pdf.

17. G-33 de Países en Desarrollo. (2005). G-33 Ministerial Communiqué, Jakarta, 12 June 2005. Extraído marzo 20, 2013, desde http://www.iatp.org/files/451_2_73101.pdf.

18. Organización Mundial del Comercio (OMC). (2010). Refocusing discussions on the Special Safeguard Mechanism (SSM): outstanding issues and concerns on its design and structure submission by the G-33 (TN/AG/GEN/30). Extraído marzo 20, 2013, desde http://ictsd.org/downloads/2010/02/g-33-ssm-paper-28jan2010.pdf.

19. International Centre for Trade and Sustainable Development (ICTD). (2010). "Negociaciones OMC se enfocan en mecanismo de salvaguardia especial y barreras no arancelarias". Puentes Quincenal, 7(3). Extraído abril 20, 2013, desde http://ictsd.org/i/news/puentesquincenal/70572/.

20. Organización Mundial del Comercio (OMC). (2010). Refocusing discussions on the Special Safeguard Mechanism (SSM): outstanding issues and concerns on its design and structure submission by the G-33 (TN/AG/GEN/30). Extraído marzo 20, 2013, desde http://ictsd.org/downloads/2010/02/g-33-ssm-paper-28jan2010.pdf.

21. Organización Mundial del Comercio (OMC). (2010). Refocusing discussions on the Special Safeguard Mechanism (SSM): outstanding issues and concerns on its design and structure submission by the G-33 (TN/AG/GEN/30). Extraído marzo 20, 2013, desde http://ictsd.org/downloads/2010/02/g-33-ssm-paper-28jan2010.pdf. 
and Sustainable Development, febrero de 2010: 1). ${ }^{22}$ En concreto, el G-33 presentó propuestas relativas al comercio estacional. Al respecto, el Grupo solicitó que se tomaran en cuenta las variaciones estacionales en la producción y el comercio, y que se revisara el lenguaje de la actual estacionalidad, pues el actual lenguaje no refleja adecuadamente los actuales patrones del comercio. Así, pidió que se distinguiera entre la "estacionalidad en comercio" y la "estacionalidad en producción", por cuanto "mientras que la temporada de cultivos podría significar que la producción de ciertos bienes esté concentrada en algunos meses particulares del año, estas tendencias no necesariamente se traducen en incrementos del comercio internacional durante esos períodos" (International Centre for Trade and Sustainable Development, febrero de 2010: 2). ${ }^{23}$ Asimismo, señalaron que las salvaguardias especiales para la agricultura, es decir, los anteriores mecanismos de salvaguardia de la OMC, "han atendido a la temporalidad pero desde la perspectiva de los importadores. Es por eso que este tipo de salvaguardia principalmente es usada por los países desarrollados para proteger a sus productores domésticos" (International Centre for Trade and Sustainable Development, febrero de 2010: 2). ${ }^{24}$

Por su parte, el G-20 ha declarado su posición respecto a la fórmula mixta para las reducciones arancelarias propuestas por la Unión Europea y Estados Unidos antes de Cancún, que "mezcla" tres fórmulas: el enfoque de la Ronda de Uruguay, que se aplica a una categoría; la fórmula de Suiza, que se aplica a otra, y una tercera, que es libre de impuestos y en la cual los productos son categorizados según su sensibilidad. ${ }^{25}$ El G-20 considera este enfoque como uno "fundamentalmente defectuoso para el acceso al mercado agrícola" (G-20 de países en desarrollo, 2005), ${ }^{26}$ ya que establece reducciones arancelarias mucho más altas para los países en desarrollo. Desde el punto de vista del G-20, la fórmula mixta "evitaría la apropiada puesta en marcha del mando de Doha para el acceso a los mercados [y] acomodaría el interés de los proponentes, en detrimento de los intereses de la mayoría de los miembros" (G-20 de países en desarrollo, 2005). ${ }^{27}$ Especialmente, las criticas que presentó el G-20 consisten en que la fórmula mixta mantendría el statu quo proteccionista, impondría una carga demasiado onerosa de reduccion arancelaria sobre los

22. International Centre for Trade and Sustainable Development (ICTD). (2010). "Negociaciones OMC se enfocan en mecanismo de salvaguardia especial y barreras no arancelarias". Puentes Quincenal, 7(3). Extraído abril 20, 2013, desde http://ictsd.org/i/news/puentesquincenal/70572/.

23. International Centre for Trade and Sustainable Development (ICTD). (2010). "Negociaciones OMC se enfocan en mecanismo de salvaguardia especial y barreras no arancelarias". Puentes Quincenal, 7(3). Extraído abril 20, 2013, desde http://ictsd.org/i/news/puentesquincenal/70572/.

24. International Centre for Trade and Sustainable Development (ICTD). (2010). "Negociaciones OMC se enfocan en mecanismo de salvaguardia especial y barreras no arancelarias". Puentes Quincenal, 7(3). Extraído abril 20, 2013, desde http://ictsd.org/i/news/puentesquincenal/70572/.

25. Para una descripción de la "fórmula mixta", véase Organización Mundial del Comercio (OMC). (n. d.). "Market access: tariffs and tariff quotas". Extraído abril 30, 2013, desde http://www.wto.org/english/tratop_e/agric_e/ negs_bkgrnd10_access_e.htm.

26. G-20 de países en desarrollo. (2005). Two Years of Activities of the G-20: Moving Forward the Doha Round. Extraído diciembre 5, 2009, desde http://www.g-20.mre.gov.br.

27. G-20 de países en desarrollo. (2005). Two Years of Activities of the G-20: Moving Forward the Doha Round. Extraído diciembre 5, 2009, desde http://www.g-20.mre.gov.br. 
países en desarrollo y permitiría a los países desarrollados proteger sus alzas arancelarias sobre productos de exportación (G-20 de países en desarrollo, 2005). ${ }^{28}$

Al respecto, el papel del G-20 fue fundamental, al resistir la imposición de la fórmula mixta y desarrollar una fórmula escalonada, ${ }^{29}$ basada en un único enfoque tanto para los países en desarrollo como para los desarrollados. Este fue el enfoque acordado en agosto de 2004 y que el G-20 consideró como el elemento central para una mejora sustancial del acceso al mercado. Según la perspectiva del Grupo, el "texto acordado suscribe los elementos clave para tal efecto, tales como la necesidad de tomar en cuenta las diferentes estructuras arancelarias, la necesidad de garantizar la seguridad alimentaria y la subsistencia de las poblaciones rurales en los países en desarrollo, y el concepto de proporcionalidad" (G-20 de países en desarrollo, 2005: 58). ${ }^{30}$

En septiembre de 2012, el G-20 presentó, dentro del proyecto de "entendimiento" (Organización Mundial del Comercio, 28 de septiembre de 2012), ${ }^{31}$ una propuesta relativa a la administración de los contingentes arancelarios ${ }^{32}$ con el propósito de facilitar el comercio y aplicar a los volúmenes comprometidos en los contingentes derechos menores a los que se aplican fuera de ellos (Organización Mundial del Comercio, 7 de diciembre de 2012), ${ }^{33}$ puesto que, según argumentan algunos países, "la administración de los contingentes [...] puede ser demasiado engorrosa y comprometer la capacidad de los exportadores para acceder a los mercados" (Organización Mundial del Comercio, 16 de noviembre de 2012). ${ }^{34}$ Esta propuesta consiste en establecer disciplinas más rigurosas para la administración de los contingentes, ya que "Ios derechos aplicados a las cantidades dentro del contingente son más bajos que los aplicados a las cantidades fuera del contingente" (Organización Mundial del Comercio, 16 de noviembre de 2012). ${ }^{35}$

28. G-20 de países en desarrollo. (2005). Two Years of Activities of the G-20: Moving Forward the Doha Round. Extraído diciembre 5, 2009, desde http://www.g-20.mre.gov.br.

29. Para una descripción de la "fórmula escalonada", véase http://www.centad.org/tariff_terminology.asp.

30. G-20 de países en desarrollo. (2005). Two Years of Activities of the G-20: Moving Forward the Doha Round. Extraído diciembre 5, 2009, desde http://www.g-20.mre.gov.br.

31. Organización Mundial del Comercio (OMC). (2012). "Según el Presidente, las propuestas del G-20 relativas a la agricultura podrían reavivar el compromiso de los negociadores con la Ronda". Extraído abril 30, 2013, desde http:/ /www.wto.org/spanish/news_s/news12_s/agng_28sep12_s.htm.

32. La 'administración de los contingentes arancelarios' se refiere a cómo se reparten entre los importadores las importaciones dentro de los contingentes cuando los derechos aplicables dentro de ellos son menores que los impuestos a las cantidades importadas fuera del contingente. Véase http://www.wto.org/spanish/news_s/news12_s/ agng_28sep12_s.htm.

33. Organización Mundial del Comercio (OMC). (2012). "El Presidente se siente alentado por la voluntad demostrada por los encargados de las negociaciones sobre la agricultura, a pesar de la preocupación que suscita la cuestión del equilibrio". Extraído abril 20, 2013, desde http://www.wto.org/spanish/news_s/news12_s/a.

34. Organización Mundial del Comercio (OMC). (2012). "El Presidente informa que no hubo una respuesta negativa pero tampoco una positiva a la propuesta relativa a las negociaciones sobre la agricultura". Extraído abril 20, 2013, desde http://www.wto.org/spanish/news_s/news12_s/agng_16nov12_s.htm.

35. Organización Mundial del Comercio (OM̄MC). (2012). "El Presidente informa que no hubo una respuesta negativa pero tampoco una positiva a la propuesta relativa a las negociaciones sobre la agricultura". Extraído abril 20, 2013, desde http://www.wto.org/spanish/news_s/news12_s/agng_16nov12_s.htm. 
China, por su parte, ha demostrado el interés que comparte con los países en desarrollo en lo relativo al acceso a los mercados, desde que inició su proceso de reforma en 1978, cuando, de forma unilateral, comenzó a aplicar a sus aranceles agrícolas reducciones profundas que se acentuaron antes y después de su ingreso a la OMC, en 2001 (Rosen, Rozelle \& Huang, 2004).

En general, en este pilar de las negociaciones agrícolas las propuestas de China han girado en torno a (1) la reducción arancelaria y la ampliación de los contingentes arancelarios en los países desarrollados, con el fin de eliminar los aranceles altos y los máximos arancelarios, y (2) el desarrollo de un trato especial y diferenciado para los países en desarrollo. Para el Gobierno chino, el único medio efectivo para proteger la agricultura son los aranceles, ya que no puede ofrecer mucha ayuda en subsidios. China ha efectuado considerables recortes arancelarios desde 1992: ha reducido los aranceles hasta en un $72 \%$, superando la reducción que han efectuado los países desarrollados (53 \%) en las rondas de Uruguay y de Doha (Zhihong, 2009).

En lo que respecta a la postura de China frente a los países desarrollados, se observa que este país desempeña un papel ofensivo (Zhihong, 2009). Debido a que el acceso de China a la $\mathrm{OMC}$, junto con la liberalización injusta del comercio, ha implicado efectos negativos para la agricultura y los agricultores chinos, este país ha sostenido que es prioritario que "los países desarrollados eliminen las distorsiones al comercio y las reglas desiguales, tales como la desigual estructura de aranceles y las diferencias en los derechos y obligaciones entre los miembros desarrollados y en desarrollo de la OMC" (Zhihong, 2009: 5).

En cambio, respecto a los países en desarrollo, China desempeña un papel defensivo, con el objetivo de minimizar el impacto de fuerzas externas en su agricultura (Zhihong, 2009). Su propuesta consiste en reducciones arancelarias moderadas, acompañadas por excepciones flexibles y salvaguardias (específicamente, la fórmula de reducción arancelaria y las implicancias de las flexibilidades, que incluyen productos especiales, productos sensibles, mecanismos de salvaguardia especial y el trato a miembros de reciente adhesión), ${ }^{36}$ y en ella las flexibilidades incluyen un margen de reducción más bajo, a excepción de ciertos productos sensibles y un período de implementación más extenso (Zhihong, 2009).

\section{Segundo pilar: ayuda interna ${ }^{37}$}

En este pilar, el G-33, con la aspiración de lograr un trato especial adicional para proteger a sus agricultores pobres, propuso que se adopten nuevas medidas para flexibilizar las disciplinas en

36. China es a la vez país en desarrollo y miembro de reciente adhesión.

37. Para obtener más detalles sobre los aspectos que cubre el pilar dedicado a la ayuda interna, véase: Organización Mundial del Comercio (OMC). (n. d.). "Agricultura: mercados más equitativos para los agricultores". Extraído marzo 25, 2013, desde http://www.wto.org/spanish/thewto_s/whatis_s/tif_s/agrm3_s.htm. 
materia de ayuda interna, con el propósito de mejorar la seguridad alimentaria mediante la concesión de ayuda a los agricultores pobres. Esta propuesta se orienta a que se relajen las disciplinas concernientes al sostenimiento de los precios en relación con la constitución de existencias públicas y la ayuda alimentaria interna de los países en desarrollo (Organización Mundial del Comercio, 27 de marzo de 2013), ${ }^{38}$ por lo que las nuevas disposiciones deberían permitir "excluir del cálculo del tipo de ayuda interna cuyo uso debe limitarse porque causa distorsión en los mercados la constitución de existencias públicas y las compras por el gobierno a los agricultores pobres a precios de sostenimiento" (Organización Mundial del Comercio, 16 de noviembre de 2012). ${ }^{39}$ Esta propuesta de constitución de existencias con fines de seguridad alimentaria en los países en desarrollo, presentada en noviembre de 2012 (Organización Mundial del Comercio, 27 de marzo de 2013), ${ }^{40}$ implica modificar la definición de las subvenciones contenidas en el Compartimento Verde (Green Box) que están permitidas sin límites debido a que su efecto de distorsión en el comercio es nulo o, como mucho, mínimo (Organización Mundial del Comercio, 16 de noviembre de 2012). ${ }^{41}$

En cuanto al G-20, su contribución a la negociación de este pilar fue decisiva para definir los recortes, las disciplinas y el monitoreo. Según el punto de vista del G-20 expresado en la Propuesta Marco en Agricultura de septiembre del 2003, "todos los países desarrollados deberían lograr reducciones sustanciales en la distorsión al comercio para que los miembros que aplican los subsidios que más distorsionan el mercado efectúen los mayores esfuerzos" (G-20 de países en desarrollo, 2005: 25)..$^{42}$ El Grupo también ha expresado sus puntos de vista acerca del Compartimento Azul, el Compartimento Verde y el Compartimento Ámbar, ${ }^{43}$ por medio de documentos informales en los que ha propuesto la forma en que los subsidios agrícolas se deberían reformar. ${ }^{44}$

38. Organización Mundial del Comercio (OMC). (2013). "Los Miembros comienzan a negociar sobre una propuesta relativa a las existencias de alimentos de los países pobres". Extraído abril 30, 2013, desde http://www.wto.org/ spanish/news_s/news13_s/agng_27mar13_s.htm.

39. Organización Mundial del Comercio (OMC). (2012). “El Presidente informa que no hubo una respuesta negativa pero tampoco una positiva a la propuesta relativa a las negociaciones sobre la agricultura". Extraído abril 20, 2013, desde http://www.wto.org/spanish/news_s/news12_s/agng_16nov12_s.htm.

40. Organización Mundial del Comercio (OMMC). (2013). "Los Miembros comienzan a negociar sobre una propuesta relativa a las existencias de alimentos de los países pobres". Extraído abril 30, 2013, desde http://www.wto.org/ spanish/news_s/news13_s/agng_27mar13_s.htm.

41. Organización Mundial del Comercio (OMC). (2012). “El Presidente informa que no hubo una respuesta negativa pero tampoco una positiva a la propuesta relativa a las negociaciones sobre la agricultura". Extraído abril 20, 2013, desde http://www.wto.org/spanish/news_s/news12_s/agng_16nov12_s.htm.

42. G-20 de países en desarrollo. (2005). Two Years of Activities of the G-20: Moving Forward the Doha Round. Extraído diciembre 5, 2009, desde http://www.g-20.mre.gov.br.

43. El Compartimento Azul, el Compartimento Verde y el Compartimento Ámbar son las categorías de los compartimentos en los que se divide el pilar de ayuda interna del Acuerdo de Agricultura de la OMC.

44. Para obtener mayor información sobre las propuestas del G-20 acerca del Compartimento Azul, el Compartimento Verde y el Compartimento Ámbar, véase la publicación Bridges Weekly Trade News Digest, del International Centre for Trade and Sustainable Development (ICTSD), en http://ictsd.org/. 
En este aspecto, China, que en comparación con muchos países desarrollados y en desarrollo no cuenta con un sistema interno formal de subsidios agrícolas bien desarrollado, aspira a acordar una mayor disciplina en las ayudas internas, particularmente de las economías desarrolladas (Rosen, Rozelle \& Huang, 2004). China propone entonces una reforma robusta tendiente a la reducción de la ayuda interna, debido a que, por ejemplo, según los términos del ingreso de China a la OMC, a este país se le permite mantener una ayuda interna equivalente al 8,5\% del valor bruto del producto agropecuario de ayuda interna (GVAO, por sus siglas en inglés), mientras que a los países en desarrollo se les permite un $10 \%$, como lo establece el Acuerdo de la Ronda de Uruguay, que fija para los países en desarrollo un $10 \%$ y para países desarrollados, un 5 \% (Rosen, Rozelle \& Huang, 2004).

De esta manera, al igual que en el pilar de acceso a los mercados, China desempeña frente a los países desarrollados un papel ofensivo, al pedir estándares estrictos sobre las provisiones del Compartimento Verde y el Comportamiento Azul para los países desarrollados, lo que sería beneficioso para los países en desarrollo. Aquí solicita recortes significativos en la medida global de ayuda (MGA) y en la ayuda comercial causante de distorsión al comercio (OTDS, por sus siglas en inglés), de los países desarrollados (Zhihong, 2009). En este aspecto, China sostiene que mayores reducciones en la ayuda de los países desarrollados mejorarían la proporción del comercio agrícola de los países en desarrollo (Zhihong, 2009).

Frente a los países en desarrollo, aquí también asume una actitud defensiva al plantear flexibilidades y excepciones con el propósito de "mantener y, si es posible, mejorar el tratamiento especial y diferenciado para los países en desarrollo, y así poder ayudar a los agricultores de bajos ingresos y de recursos escasos" (Zhihong, 2009: 4). Asimismo, sostiene que "los países en desarrollo no deberían estar sujetos a los límites de los subsidios del Compartimento Azul, y los requisitos de gastos del Compartimento Verde deberían considerar las circunstancias especiales de los países en desarrollo" (Zhihong, 2009: 4).

\section{Tercer pilar: subsidio a las exportaciones ${ }^{45}$}

Aunque el G-33 se ha concentrado más bien en propuestas concernientes a los dos pilares anteriores, el G-20 ha presentado iniciativas en los tres, planteando en el tercero de ellos la eliminación de los subsidios a las exportaciones para los productos de particular interés para los países en desarrollo. En 2007, "el Grupo hizo un Ilamado para que se hicieran 'recortes reales y efectivos' aplicables a los subsidios agrícolas, recalcando que no permitirían que Estados Unidos

45. Para obtener más detalles sobre los aspectos que cubre el pilar dedicado a los subsidios a las exportaciones, véase Organización Mundial del Comercio (OMC). (n. d.). "Agricultura: mercados más equitativos para los agricultores". Extraído marzo 25, 2013, desde http://www.wto.org/spanish/thewto_s/whatis_s/tif_s/agrm3_s.htm. 
se saliera con la suya fijando subsidios muy por encima de los niveles de gasto actuales" (International Centre for Trade and Sustainable Development, 2007). ${ }^{46}$

En este aspecto de las negociaciones agrícolas, China aspira a que los subsidios a las exportaciones agrícolas sean rápidamente eliminados, sobre la base de que -como lo permite el Acuerdo de Agricultura de la Ronda de Uruguay- Estados Unidos, la Unión Europea, Australia y Canadá, entre otros, mantuvieron su derecho a aplicar subsidios a las exportaciones (Rosen, Rozelle \& Huang, 2004). Frente a esta situación, los países en desarrollo desean mantener sus exenciones especiales y que se disminuyan los derechos que poseen las economías desarrolladas para usar subvenciones a las exportaciones (Rosen, Rozelle \& Huang, 2004). Desde su ingreso a la OMC, China dejó demostrado su interés en la eliminación de los subsidios a las exportaciones agrícolas, al eliminar sus propios subsidios a las exportaciones: "China fue más allá de lo que otras economías en desarrollo habían hecho en la Ronda de Uruguay y más allá de lo que se esperaba de las negociaciones de la Ronda de Doha" (Rosen, Rozelle \& Huang, 2004: 11).

\subsection{LIDERAZGO COLECTIVO DEL G-20 Y EL G-33: SU CAPACIDAD DE CONSENSO}

La tercera condicionante de la participación de China en estas coaliciones es el liderazgo colectivo de negociación institucional que estas han desarrollado, permitiendo así a China y los países latinoamericanos posicionar sus intereses en las negociaciones agrícolas de la OMC. En este análisis se parte de la base de que la diferencia entre el mundo desarrollado y el mundo en desarrollo no es únicamente el poder relativo que posean, sino también su poder de negociación; en consecuencia, tanto para China como para los países en desarrollo y, dentro de ellos, los latinoamericanos, es decisiva la conjunción de capacidades colectivas que les permitan aumentar su poder de negociación institucional en instancias como las rondas de negociaciones de la OMC.

Como señala la literatura al respecto, por medio del liderazgo colectivo Estados pequeños y más débiles pueden aumentar su poder de negociación institucional (Young, 1991); sin embargo, aquí se añade que no solo los Estados de menor tamaño, sino también los países en desarrollo y las potencias medianas emergentes, pueden lograr mejorar esta capacidad, por ejemplo, en el marco de las negociaciones comerciales de la OMC, como China, y otras latinoamericanas, como Brasil y México, que forman parte ya sea del G-20 o del G-33 o se encuentran en ambas, como es el caso de China. El liderazgo colectivo de ambas coaliciones se observa en el constante trabajo de presentación de propuestas relativas a las reformas a temáticas conflictivas del

46. International Centre for Trade and Sustainable Development. (2007). "G-20, G-33 Ministers Underline Priorities Before 'Decisive Phase' in Doha Talks". Bridges Weekly Trade News Digest, 11(21). Extraído diciembre 5, 2009, desde http://ictsd.org/i/news/bridgesweekly/7602/.

EL MULTILATERALISMO AD HOC EN LAS RELACIONES CHINA-AMÉRICA LATINA Y EL CARIBE Y LAS COALICIONES SUR-SUR EN LAS NEGOCIACIONES DE LA OMC SOBRE EL COMERCIO AGRÍCOLA 
comercio agrícola internacional, en representación de los intereses de países en desarrollo y subdesarrollados, así como también de los agricultores de escasos recursos, gestando diálogos con otros grupos de negociaciones y con miembros individuales.

Este liderazgo colectivo, concebido como un liderazgo basado en el consenso entre un grupo más grande de actores (Dobson, 2001), puede desplegarse en una esfera tanto interna o intragrupal como externa intergrupal con otras coaliciones. Por esfera externa se entiende la contribución de la coalición a facilitar las negociaciones en foros multilaterales (Diego-Fernández, 2008). En este nivel, el G-20 demuestra su capacidad de construcción de consenso en las relaciones que ha establecido con otros grupos dentro de la $\mathrm{OMC}$, por ejemplo con el Grupo de Cairns, del que la mayoría de los países son miembros. A pesar de estas diferencias, ambos grupos han cooperado y no han arriesgado la identidad del Grupo, sobre la base de la coincidencia de sus respectivas agendas y los intereses respecto de la necesidad de terminar con las políticas que distorsionan el comercio agrícola y la apertura de los mercados de países desarrollados (Da Motta Veiga, 2005).

En el caso del G-33, se observan en sus declaraciones sus esfuerzos por fomentar la cooperación intergrupal dentro del contexto de las negociaciones comerciales de la OMC con otras agrupaciones y actores negociadores formados por países en desarrollo, entre ellos el ACP, el Grupo Africano, los Países Menos Adelantados y, por supuesto, el G-20, señalando que "es únicamente a través de un espíritu colectivo y de cercana cooperación entre países en desarrollo que la dimensión sobre el desarrollo de la Declaración de Doha se puede lograr efectivamente" (G-33 de países en desarrollo, 12 de junio de 2005: 4). ${ }^{47}$

Otro ejemplo que evidencia la cooperación y coordinación intergrupal corresponde a la declaración conjunta acerca de las negociaciones agrícolas de la OMC publicada en diciembre de 2005 por las principales coaliciones de países en desarrollo, tales como el G-20, el G-33, el ACP, los Países Menos Adelantados, el Grupo Africano y las Economías Pequeñas. Se trata de la primera vez que estas agrupaciones negociadoras sostuvieron una reunión ministerial con el propósito de "coordinar sus esfuerzos para desarrollar un enfoque común frente a los asuntos de interés común" (International Centre for Trade and Sustainable Development, 16 de diciembre de 2005: 1), ${ }^{48}$ recalcando que es la agricultura lo central para el desarrollo y reafirmando las demandas de las economías pequeñas y vulnerables, así como las de los Países Menos Adelantados.

En la esfera interna de la cohesión, el G-33 ha demostrado un trabajo constante en la presentación de sus consecutivas propuestas y de críticas respecto de los textos emanados de las negociaciones.

47. G-33 de países en desarrollo. (2005). G-33 Ministerial Communiqué, Jakarta, 12 June 2005. Extraído marzo 20, 2013, desde http://www.iatp.org/files/451_2_73101.pdf.

48. International Centre for Trade and Sustainable Development (ICTSD). (2005). Joint Statement by G20, G33, LDC, ACP and African Group on coordination efforts. Extraído marzo 20, 2013, desde http://ictsd.org/i/wto/15453/. 
Así lo demuestran sus declaraciones emitidas públicamente, al menos durante los años 2005, 2007, 2008, 2009 y 2010, ${ }^{49}$ así como las propuestas publicadas en la prensa especializada en materia agrícola internacional. ${ }^{50} \mathrm{Al}$ mismo tiempo, en esas declaraciones se observa la actitud que el Grupo ha mantenido, expresando de forma taxativa las críticas a los borradores presentados. Así, por ejemplo, "el G-33 considera que el contenido del borrador sobre el mecanismo de salvaguardia especial es extremadamente inadecuado. Las estructuras propuestas sobre el mecanismo de salvaguardia especial solo ofrecerían a la larga un mecanismo estricto, restrictivo, gravoso, inefectivo y no operacional para los países en desarrollo y los países menos desarrollados. Además, el borrador sobre el mecanismo de salvaguardia especial también sugiere un nuevo tipo de diferenciación de tratos entre los países en desarrollo. Esto es simplemente inaceptable" (Declaración del G-33 COA-SS Informal Open Ended, febrero 2008). ${ }^{51}$ También en la declaración de mayo de 2008 el G-33 expuso su preocupación por los desequilibrios que persistieron en el borrador del texto sobre modalidades agrícolas, que no incorpora elementos fundamentales específicos de los productos especiales y el MSE (Declaración del G-33 COA-SS Informal Open Ended, mayo 2008)..$^{52}$

En el caso del G-20, esta coalición ha probado ser capaz no solo de construir consenso, sino también de mantener la cohesión interna, reuniendo a países en desarrollo que tradicionalmente adoptaron posiciones diferenciadas y opuestas en las negociaciones de la OMC. El G-20 ha procurado acomodar las diferentes preocupaciones de sus miembros, con el fin de tener una base de negociación común e inclusiva que garantice un resultado balanceado. Este espíritu constructivo ha permitido a la coalición crear consenso entre los países en desarrollo con intereses heterogéneos y elaborar una posición sólida y sustantiva al abordar los asuntos complejos que comprometen las negociaciones agrícolas (Da Motta Veiga, 2005).

Su cohesión se observa, por ejemplo, incluso antes de la Conferencia Ministerial de Cancún, cuando "algunos países desarrollados trataron de desestimar al Grupo, al rechazar aceptar sus propuestas y al acusar al Grupo de intentar introducir una dimensión ideológica en la negociación, trayendo así a la OMC posiciones y tácticas que tenían su origen en el diálogo norte-sur" (Da Motta Veiga, 2005). Otro intento de algunos países desarrollados por dividir la coalición tuvo lugar en Cancún, cuando el G-20 se establecía. Dichos países trataron de poner obstáculos a las relaciones del G-20 con otras agrupaciones dentro de la OMC, especialmente con el

49. Correspondiente al documento Refocusing Discussions on the Special Safeguard Mechanism (SSM): Outstanding Issues and Concerns on its Design and Structure Submission by the G-33, del 28 de enero de 2010.

50. Específicamente los archivos de prensa de las siguientes fuentes: International Centre for Trade and Sustainable Development (ICTSD) (http://ictsd.org/ ), Instititute for Agriculture and Trade Policy (IATP) (http://www.iatp.org/) y OMC (http://www.wto.org/spanish/news_s/archive_s/ag_arc_s.htm).

51. G-33 de países en desarrollo. (2008). G-33 Statement COA-SS Informal Open Ended, 15 February 2008. Extraído marzo 20, 2013, desde http://www.iatp.org/files/451_2_101658.pdf.

52. G-33 de países en desarrollo. (2008). G-33 Statement COA-SS Informal Open Ended, 26 May 2008. Extraído marzo 20, 2013, desde http://www.iatp.org/files/451_2_102829.pdf. 
Grupo de Cairns y el Grupo Africano (Da Motta Veiga, 2005). Como resultado de todas las presiones sobre sus miembros, una delegación se retiró del Grupo y un pequeño número de países dejó la coalición; no obstante, el G-20 permaneció unido durante toda la conferencia, e incluso la delegación de Nigeria se unió en las etapas finales de la reunión, y otros países, como Tanzania y Zimbabue, se convirtieron en miembros (Da Motta Veiga, 2005).

El G-20 ha demostrado ser capaz de ejercer gran influencia en las negociaciones agrícolas de la OMC, gozando de una gran legitimidad; ello, sobre la base de la presentación de propuestas que representan los intereses de los países en desarrollo y la formulación de críticas en temas decisivos de las negociaciones, tales como la ayuda interna, el acceso a mercados y los subsidios a las exportaciones, hasta llegar a ser considerado como el "centro de gravedad de equilibrio en las negociaciones agrícolas" (International Centre for Trade and Sustainable Development, 2007). ${ }^{53}$ De hecho, en el área agrícola, que es la más controversial de la negociación, la coalición ha logrado demandar la eliminación de los subsidios a las exportaciones sin juzgar de antemano el resultado, asegurando posteriormente el compromiso y estableciendo la fecha de su eliminación (Diego-Fernández, 2008).

En consecuencia, esta forma de funcionar de ambas coaliciones, no solo en la esfera interna, sino también en la externa, sobre la base de la construcción de consenso, permite respetar nuevos valores y los diversos intereses nacionales de los países involucrados, así como definir objetivos acordados en común, demostrados en la serie de declaraciones oficiales publicadas y el análisis de la literatura revisada. Vista entonces la capacidad de consenso intragrupal e intergrupal de ambas coaliciones, es posible comprender la participación activa de China en este tipo de organizaciones multilaterales, donde: predominan normas y reglas informales de comportamiento para sus países miembros, así como ciertos principios tales como la inclusión, la horizontalidad, la flexibilidad, la transparencia y el entendimiento mutuo. ${ }^{54}$ Por consiguiente, esta forma de operar de estas coaliciones tiende a acomodarse con el así llamado "multilateralismo cualitativo" de China (Wang, 2000), según el cual la política exterior china posee una naturaleza independiente de la occidental, en la que los principios del multilateralismo consisten en la igualdad, la confianza, la seguridad común, la no especificidad del enemigo y la resolución pacífica de los conflictos. Más bien, el énfasis de la política exterior china "está puesto en los principios de la soberanía estatal, el respeto a la diversidad política, económica y militar entre los diferentes países, y la importancia de las consultas informales y la construcción de consenso" (Wang, 2000: 479).

53. International Centre for Trade and Sustainable Development. (2007). "G-20, G-33 Ministers Underline Priorities Before 'Decisive Phase' in Doha Talks". Bridges Weekly Trade News Digest, 11(21). Extraído diciembre 5, 2009, desde http://ictsd.org/i/news/bridgesweekly/7602/.

54. El G-33 expresa reiteradamente en sus declaraciones oficiales la necesidad de que el proceso de negociación y los textos emitidos sean producto y reflejo de un enfoque bottom-up basado en la inclusión y la horizontalidad, así como también en la flexibilidad, la transparencia y el entendimiento mutuo que permitan producir textos más balanceados para los grupos negociadores y otorgarles así mayor legitimidad a los resultados del proceso negociador. Fuente: G-33 de países en desarrollo. (2007). "G-33 Ministerial Press Statement, Davos, 26 January 2007". Extraído marzo 20, 2013, desde http://www.iatp.org/files/451_2_97175.pdf. 


\section{CONCLUSIONES}

Según se demostró en este artículo, el involucramiento de China en el G-20 y el G-33 se explica por tres condicionantes: reunir a casi toda América Latina y el Caribe; la confluencia de intereses y objetivos entre ambas coaliciones y China, y la alta capacidad de consenso de ambas coaliciones. No obstante, a las tres anteriores se podría agregar una cuarta condicionante, vinculada con las características esenciales de estas instancias informales de negociación multilateral, que por su naturaleza facilitan el acercamiento y el dialogo directo y ajeno a normativas propias de las organizaciones multilaterales con alto grado de institucionalización. Según Wang (2000), la informalidad de las coaliciones se ajusta perfectamente a los principios de la política exterior china, en especial a las consultas informales y la construcción de consenso. Así, la inserción internacional de China en organizaciones multilaterales ad hoc se condice con la estrategia china de poder blando que acompaña a la diplomacia china en su búsqueda de materia prima, recursos naturales y mercados para sus exportaciones en la región, lo que viene a complementar la 'diplomacia económica', basada en la asistencia, la inversión y otros instrumentos, y a la estrategia política basada en el establecimiento de alianzas estratégicas con sus socios latinoamericanos. En este sentido, instancias de negociación colectiva como estas agrupaciones permiten a China participar en un formato de toma de decisiones que se acomoda al estilo diplomático chino, inclinado más bien al contacto cara a cara, la construcción de consensos y la resolución de controversias por medio de "mecanismos de mediación menos formales y que protejan las apariencias" (Wang, 2000: 482), pues, como afirma Wang (2000: 482), para el punto de vista chino, las instituciones formales tienden a "fomentar la imposición de las preferencias de algunos países sobre otros".

\section{BIBLIOGRAFÍA}

- $\quad$ Constantini, V., Crescenzi, R., De Filippis, F., \& Salvatici, L. (2007). "Bargaining Coalitions in the WTO Agricultural Negotiations". The World Economy, 30(5), 863-891.

- Da Motta Veiga, P. (2005). "Brazil and the G-20 Group of Developing Countries". En P. Gallagher, P. Low, \& A. L. Stoler (eds.), Managing the Challenges of WTO Participation. 45 Case Studies. Cambridge University Press.

- Diego-Fernández, M. (2008). "Trade Negotiations Make Strange Bedfellows". World Trade Review, 7(2), 423-453.

- Dobson, W. (2001). Broadening Participation in G-7 Summits. Extraído diciembre 10, 2009, desde Rotman School of Management of University of Toronto: http:// www.rotman.utoronto.ca/dobson/research/G-20\%20proposal\%202002.PDF.

- G-20 de países en desarrollo. (2005). Two Years of Activities of the G-20: Moving Forward the Doha Round. Extraído diciembre 5, 2009, desde http://www.g-20.mre.gov.br. 
- G-33 de países en desarrollo. (2005). G-33 Ministerial Communiqué, Jakarta, 12 June 2005. Extraído marzo 20, 2013, desde http://www.iatp.org/files/451_2_73101.pdf.

- G-33 de países en desarrollo. (2007). G-33 Ministerial Press Statement, Davos, 26 January 2007. Extraído marzo 20, 2013, desde http://www.iatp.org/files/451_2_97175.pdf.

- G-33 de países en desarrollo. (2008). G-33 Statement COA-SS Informal Open Ended, 15 February 2008. Extraído marzo 20, 2013, desde http://www.iatp.org/files/ 451_2_101658.pdf.

- G-33 de países en desarrollo. (2008). G-33 Statement COA-SS informal Open Ended, 26 May 2008. Extraído marzo 20, 2013, desde http://www.iatp.org/files/451_2_102829.pdf.

- International Centre for Trade and Sustainable Development. (2007). "G-20, G-33 Ministers Underline Priorities Before 'Decisive Phase' in Doha Talks". Bridges Weekly Trade News Digest, 11(21). Extraído diciembre 5, 2009, desde http://ictsd.org/i/news/bridgesweekly/7602.

- International Centre for Trade and Sustainable Development (ICTD). (2010). "Negociaciones $\mathrm{OMC}$ se enfocan en mecanismo de salvaguardia especial y barreras no arancelarias". Puentes Quincenal, 7(3). Extraído abril 20, 2013, desde http://ictsd.org/i/news/ puentesquincenal/70572/.

- International Centre for Trade and Sustainable Development (ICTSD). (2005). Joint Statement by G20, G33, LDC, ACP and African Group on coordination efforts. Extraído marzo 20, 2013, desde http://ictsd.org/i/wto/15453/.

- Ismail, F. (2007). The G-20 and NAMA 11: The Role of Developing Countries in the WTO Doha Round. Seminar Series on Making Globalization Work for Developing Countries, Global Economic Governance Programme.

- Jales, M. (2005). Tariff Reduction, Special Products and Special Safeguards: An Analysis of the Agricultural Tariff Structures of G-33 Countries. Ginebra: International Centre for Trade and Sustainable Development (ICTSD).

- $\quad$ Organización Mundial del Comercio (OMC). (2012). “El Presidente informa que no hubo una respuesta negativa pero tampoco una positiva a la propuesta relativa a las negociaciones sobre la agricultura". Extraído abril 20, 2013, desde http://www.wto.org/spanish/news_s/ news12_s/agng_16nov12_s.htm.

- $\quad$ Organización Mundial del Comercio (OMC). (2013). "Los Miembros comienzan a negociar sobre una propuesta relativa a las existencias de alimentos de los países pobres". Extraído abril 30, 2013, desde http://www.wto.org/spanish/news_s/news13_s/ agng_27mar13_s.htm.

- $\quad$ Organización Mundial del Comercio (OMC). (2010). Refocusing discussions on the Special Safeguard Mechanism (SSM): outstanding issues and concerns on its design and structure submission by the G-33 (TN/AG/GEN/30). Extraído marzo 20, 2013, desde http://ictsd.org/ downloads/2010/02/g-33-ssm-paper-28jan2010.pdf.

- Organización Mundial del Comercio (OMC). (2012). "El Presidente se siente alentado por la voluntad demostrada por los encargados de las negociaciones sobre la agricultura, a pesar de la preocupación que suscita la cuestión del equilibrio". Extraído abril 20, 2013, desde http://www.wto.org/spanish/news_s/news12_s/a. 
- $\quad$ Organización Mundial del Comercio (OMC). (2012). "Según el Presidente, las propuestas del G-20 relativas a la agricultura podrían reavivar el compromiso de los negociadores con la Ronda". Extraído abril 30, 2013, desde http://www.wto.org/spanish/news_s/news12_s/ agng_28sep12_s.htm.

- $\quad$ Organización Mundial del Comercio (OMC). (n. d.). "Agricultura: mercados más equitativos para los agricultores". Extraído marzo 25, 2013, desde http://www.wto.org/spanish/ thewto_s/whatis_s/tif_s/agrm3_s.htm.

- Organización Mundial del Comercio (OMC). (n. d.). "Grupos en el marco de las negociaciones". Extraído abril 30, 2013, desde http://www.wto.org/spanish/tratop_s/dda_s/ negotiating_groups_s.htm.

- Organización Mundial del Comercio (OMC). (n. d.). "Grupos en las negociaciones sobre la agricultura". Extraído abril 30, 2013, desde http://www.wto.org/spanish/tratop_s/agric_s/ negoti_groups_s.htm.

- Organización Mundial del Comercio (OMC). (n. d.). "Market access: tariffs and tariff quotas". Extraído abril 30, 2013, desde http://www.wto.org/english/tratop_e/agric_e/ negs_bkgrnd10_access_e.htm.

- Rosales, O. (2009). "La globalización y los nuevos escenarios del comercio internacional". Revista Cepal (97), 77-95.

- $\quad$ Rosen, D., Rozelle, S., \& Huang, J. (2004). Roots of Competitiveness: China's Evolving Agriculture Interests. Peterson Institute for International Economics. Extraído marzo, 2013, desde http://bookstore.piie.com/book-store/380.html.

- $\quad$ Scott, J., \& Wilkinson, R. (2011). China and the WTO. Indiana University, Research Center for Chinese Politics and Business.

- Wang, H. (2000). "Multilateralism in Chinese Foreign Policy: The Limits of Socialization". Asian Survey, 4(3), 475-491.

- Wu, G., \& Lansdowne, H. (2008). China Turns to Multilateralism: Foreign Policy and Regional Security. Londres y Nueva York: Routledge.

- Young, O. R. (1991). "Political Leadership and Regime Formation: On the Development of Institutions in International Society". International Organization, 45(3), 281-208.

- Zhihong, T. (2009). Implications for China of the December 2008 Draft Agricultural Modalities. Ginebra: International Centre for Trade and Sustainable Development (ICTSD).

- Zhu, Z. (2010). China's New Diplomacy: Rationale, Strategies and Significance. Farnham: Ashgate. 